\title{
Does time equal vision in the acute treatment of a cohort of AQP4 and MOG optic neuritis?
}

Hadas Stiebel-Kalish, MD, Mark Andrew Hellmann, MD, Michael Mimouni, MD, Friedemann Paul, MD, Omer Bialer, MD, Michael Bach, PhD, and Itay Lotan, MD

Neurol Neuroimmunol Neuroinflamm 2019;6:e572. doi:10.1212/NXI.0000000000000572

\section{Abstract}

\section{Objective}

To investigate whether visual disability which is known to accumulate by poor recovery from optic neuritis (ON) attacks can be lessened by early treatment, we investigated whether the time from symptom onset to high-dose IV methylprednisolone (IVMP) affected visual recovery.

\section{Methods}

A retrospective study was performed in a consecutive cohort of patients following their first aquaporin-4 (AQP4)-IgG or myelin oligodendrocyte glycoprotein (MOG)-IgG-ON. Bestcorrected visual acuity (BCVA) in ON eyes at 3 months (BCVA3mo) was correlated with time to IVMP (days). In cases of bilateral ON, 1 eye was randomly selected.

\section{Results}

A total of 29 of 37 patients had ON (27 AQP4-seropositive neuromyelitis optica spectrum disorder [NMOSD] and 9 MOG-IgG-ON), 2 of whom refused treatment. Of the 27 patients included, 10 presented later than 7 days from onset. The median BCVA3mo of patients treated $>7$ days was 20/100 (interquartile range 20/100-20/200). Patients treated $>7$ days had an OR of 5.50 (95\% CI 0.88-34.46, $p=0.051$ ) of failure to regain $0.0 \operatorname{logMAR}$ vision $(20 / 20)$ and an OR of 10.0 (95\% CI 1.39-71.9) of failure to regain $0.2 \log$ MAR vision $(20 / 30)(p=0.01)$ compared with patients treated within 7 days. ROC analysis revealed that the optimal criterion of delay in IVMP initiation was $\leq 4$ days, with a sensitivity and specificity of $71.4 \%$ and $76.9 \%$, respectively.

\section{Conclusions}

In this retrospective study of ON with AQP4 and MOG-IgG, even a 7-day delay in IVMP initiation was detrimental to vision. These results highlight the importance of early treatment for the long-term visual recovery in this group of patients. A prospective, multicenter study of the effects of timing of IVMP is currently underway.

\section{Classification of evidence}

This study provides Class IV evidence that hyperacute treatment of AQP4 and MOG-ON with IVMP increases the chance for good visual recovery (20/20 vision) and that even a greater than 7 -day delay in treatment is associated with a higher risk for poor visual recovery.

\author{
Correspondence \\ Dr. Stiebel-Kalish \\ kalishhadas@gmail.com
}

\section{MORE ONLINE}

$\rightarrow$ Class of Evidence

Criteria for rating

therapeutic and diagnostic

studies

NPub.org/coe

From the Sackler School of Medicine (H.S.-K., M.A.H., O.B., I.L.), Tel Aviv University; Neuro-Ophthalmology Unit (H.S.-K., O.B.), Department of Ophthalmology, Rabin Medical Center; Neuro-Immunology Service and Department of Neurology (M.A.H., I.L.), Rabin Medical Center, Petah Tikva; Department of Ophthalmology (M.M.), Rambam Health Care Campus, and Ruth Rappaport Faculty of Medicine, Technion-Israel Institute of Technology, Haifa, Israel; NeuroCure Clinical Research Center and Experimental and Clinical Research Center (F.P.), Max Delbrueck Center for Molecular Medicine, Charité-Universitätsmedizin Berlin, Corporate Member of Freie Universität Berlin, Humboldt-Universität zu Berlin, and Berlin Institute of Health; and Eye Center (M.B.), Medical Center, University of Freiburg and Faculty of Medicine, University of Freiburg, Germany. 


\section{Glossary}

AON = autoimmune optic neuritis; AQP = aquaporin; AUC = area under the curve; $\mathbf{B C V A}=$ best-corrected visual acuity; IA = immunoadsorption; IVMP = IV methylprednisolone; $\mathbf{M O G}=$ myelin oligodendrocyte glycoprotein; $\mathbf{O C T}=$ optical coherence tomography; $\mathbf{O N}$ = optic neuritis; ONTT = Optic Neuritis Treatment Trial; PE = plasma exchange; RGC = retinal ganglion cell; VA = visual acuity.

\section{Is visual recovery in optic neuritis dependent on the timing of treatment?}

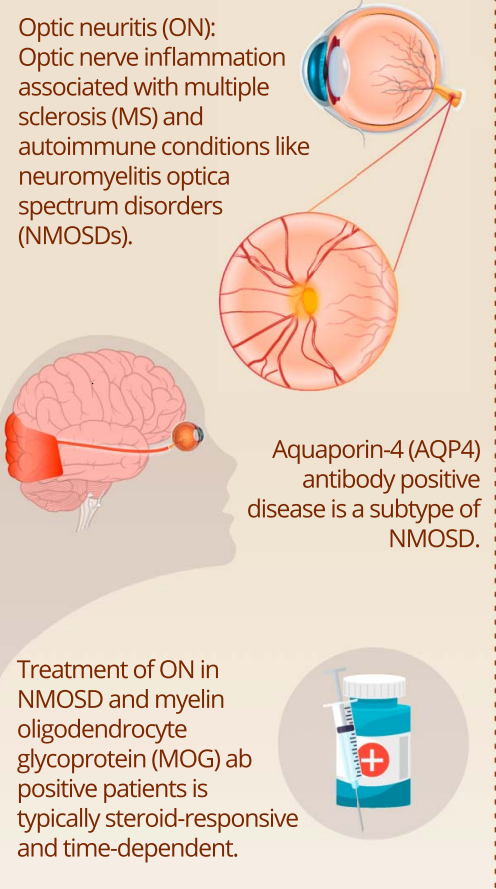

doi:10.1212/NXI.0000000000000572

Copyright (c) 2019 American Academy of Neurology
Patient cohort:

27 AQP4-seropositive

NMOSD

9 MOG-IgG positive ON

Does the timing

of intravenous

methyl-

prednisolone

(IVMP) treatment

affect visual

outcomes in

AQP4-IgG and

MOG-IgG

positive patients with ON?

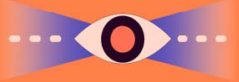

Treatment:

$1000 \mathrm{mg}$ IVMP for 3-5 days, Best-corrected visual acuity (BCVA) at 3 months. then oral prednisone

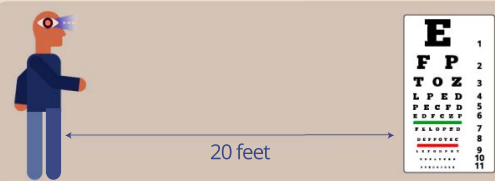

Patients with AQP4- and MOG-positive ON responded better to earlier IVMP treatment.

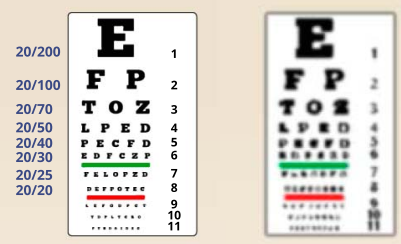

Patients treated later than $\mathbf{4}$ days

had an odds ratio of 8.33 of failure to regain $\mathbf{2 0 / 2 0}$ vision.

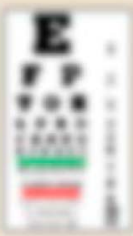

Patients treated later than $\mathbf{7}$ days

had odds ratio of 10.0 of failure to regain $\mathbf{2 0 / 3 0}$ vision.

Early treatment is essential for long-term visual recovery in this group of patients.

Even a 7-day delay in IVMP initiation was detrimental to the vision of patients with AQP4 and MOG-IgG ON.
Optic neuritis $(\mathrm{ON})$ is a common inflammation of the optic nerve associated with numerous autoimmune conditions, including MS, neuromyelitis optica spectrum disorders (NMOSDs), chronic relapsing inflammatory optic neuritis (CRION), and autoimmune optic neuritis (AON). ${ }^{1-5}$ NMOSD is further subdivided into aquaporin-4 (AQP4) antibody-positive disease and a seronegative form. ${ }^{6}$ A subset of patients with $\mathrm{ON}$ have serum IgG autoantibodies to myelin oligodendrocyte glycoprotein (MOG). ${ }^{7-11}$ The protein and cellular targets of these 2 antibodies are distinct in that AQP4 is expressed on astrocytes and retinal Müller cells, whereas MOG is expressed by oligodendrocytes. ${ }^{12,13}$ Despite these pathogenic differences, $\mathrm{ON}$ attacks in both conditions are treated similarly with high-dose corticosteroids and/or plasma exchange (PE). Although some patients with MOG ab disease meet the 2015 criteria for NMOSD, there is an ongoing debate as to whether MOG ab-positive patients should receive a diagnosis of NMOSD. ${ }^{14}$ Although a significant number of MOG ab-positive patients have a relapsing course leading to accumulative disability, others do not relapse; thus, their inclusion together with other AQP4seronegative patients with NMOSD could compromise the study of therapeutic candidates in NMOSD. ${ }^{14}$

Acute treatment of $\mathrm{ON}$ in MS was shaped by the North American Optic Neuritis Treatment Trial (ONTT), which showed that IV methylprednisolone (IVMP) accelerates recovery but does not affect the final visual outcome. ${ }^{15,16}$

However, the clinical course of ON in NMOSD and in MOG ab-positive patients differs from MS and is typically steroid responsive or dependent. Disability from both AQP4 and MOG$\mathrm{ON}$ is accumulated by poor recovery from attacks. ${ }^{17}$ The recommended acute treatment options in antibody-mediated $\mathrm{ON}$ are high-dose IVMP, PE, and immunoadsorption. ${ }^{18,19}$

Historically, NMOSD-ON has been associated with a poor visual outcome. $^{20}$ Studies have correlated the visual outcome of AQP4$\mathrm{ON}$ attacks with the severity of visual loss at presentation, type of antibody, and with the use of additional PE. ${ }^{21,22}$ Visual disability 
has been shown to be accrued with each attack, resulting in poor quality of life. ${ }^{13}$ Three previous studies focused on the effect of timing of IVMP on visual outcome. ${ }^{23-25}$ These studies included several subtypes of ON, with only a few patients with NMOSD and no MOG-positive patients.

In this study, we tested the hypothesis that timing of IVMP affects visual outcome in a cohort of AQP4-IgG and MOGIgG-positive patients with $\mathrm{ON}$ by analyzing the effect of the number of days until treatment commenced with the bestcorrected visual acuity (BCVA) at 3 months.

\section{Methods}

\section{Patients}

We conducted a retrospective case review of a cohort of all consecutive patients presenting to a tertiary referral neuroophthalmology and neuroimmunology center at Rabin Medical Center, Israel, with a first event of AQP4 or MOGON between January 2005 and June 2018.

\section{Standard protocol approvals, registrations, and patient consents}

The study was performed following IRB approval in accordance with the World Medical Association Declaration of Helsinki. The neuro-ophthalmology unit database was searched for the diagnoses of NMOSD, AQP4, and MOGassociated $\mathrm{ON}$.

\section{Inclusion and exclusion criteria}

$\mathrm{ON}$ was diagnosed based on a combination of clinical history, objective findings as determined by clinical examination of a neuro-ophthalmologist, and paraclinical tests. These included patients presenting with subacute onset vision loss, pain with eye movement, visual field defects consistent with an optic nerve injury, color defects, MRI evidence of optic nerve inflammation (increased T2 signal, gadolinium enhancement, and optic nerve swelling), ${ }^{26}$ and neurophysiologic abnormalities (delayed visual evoked potential latencies). ${ }^{27}$ Exclusion criteria were other ocular causes of poor visual acuity (VA) and treatment refusal. This retrospective cohort study focused on VA as a functional outcome and did not examine other functional parameters such as visual field or structural-anatomic outcome measures such as optical coherence tomography (OCT) outcomes because for some patients, these were either missing (3 patients) or performed by different machines ( 2 patients).

Patients had to have a diagnosis of NMOSD, AQP4, or MOGassociated $\mathrm{ON}$ based on established diagnostic criteria. ${ }^{6,28}$ AQP4 antibodies were tested using a commercial cell-based kit (EUROIMMUN, Lübeck, Germany). In addition, AQP4 IgG antibodies were tested at the Center for Autoimmune Neurology in Barcelona, Spain, using tissue immunohistochemistry and cell-based assays. ${ }^{28,29}$ MOG-IgG antibodies were tested by cell-based assays at the Center for Autoimmune Neurology in Barcelona, Spain. ${ }^{30}$

\section{Treatment}

The treatment received was IVMP at a daily dose of $1,000 \mathrm{mg}$ for 3-5 days, followed by oral prednisone (starting at $1 \mathrm{mg}$ / $\mathrm{kg} / \mathrm{d}$ ). At the time of presentation, antibody status was not known for the majority of patients, but oral prednisone treatment was prolonged in patients with relapse of visual loss following steroid cessation or in patients presenting with clinical or paraclinical findings suggestive of AQP4 or MOG antibody disease. Patients who refused acute treatment with IVMP for ON were excluded from this study (figure e-1, flowchart, links.lww.com/NXI/A116).

\section{Clinical assessment and medical notes}

Medical notes had to include a detailed report of the timing of patient-reported onset of visual loss, timing of IVMP treatment, and documentation of high-contrast BCVA examination in each eye at 3 months following the attack.

\section{Main outcome measures}

The main outcome measure of this study was 3-month BCVA. Secondary outcomes were failure to regain $0.0 \operatorname{logMAR}(20 /$ 20) and 0.2 logMAR vision (20/30) vision at the 3 -month follow-up visit.

\section{Level of evidence}

This is a level IV retrospective cohort study comparing the BCVA at 3 months of patients with AQP4 and MOG-ON presenting early for IVMP treatment vs those patients presenting late.

\section{Statistical analysis}

Descriptive statistics were calculated using SAS software (v9.4). Median logMAR BCVA at 3 months ("BCVA3mo") and interquartile ranges were documented at 3 months. Patients were grouped according to BCVA3mo into those achieving 0.0 $\operatorname{logMAR}(20 / 20)$ vision and those whose BCVA3mo was worse than 0.0 logMAR. Outcome was correlated with time (in days) from symptom onset to IVMP ("time to IVMP"). For patients with bilateral ON, 1 eye was randomly included in the analysis. A receiver operator curve (ROC) was used to analyze the best sensitivity and specificity using the Youden index ${ }^{31}$ for the best cutoff time to IVMP to achieve the best BCVA3mo. The relative risk, OR, and confidence intervals for BCVA3mo worse than 0.0 $\log$ MAR (20/20) and 0.2 logMAR vision (20/30) were analyzed for patients treated early (time to IVMP $\leq 6$ days) compared with patients treated after day 7 . Two-tailed tests were used, and $p<0.05$ was accepted as statistically significant.

\section{Data availability}

Data have been uploaded and will be made readily available upon publication at the following Mendeley data repository: dx.doi.org/10.17632/ht5s9cc845.1.

\section{Results}

Thirty-seven patients were enrolled. Twenty-eight patients fulfilled the 2015 diagnostic criteria ${ }^{6}$ for NMOSD (27 were AQP4 positive, and 1 patient was seronegative), and another 9 
had MOG-IgG positive ON. Included in this study were 27 AQP4-positive and 9 MOG-positive patients with ON. Figure e-1 (links.lww.com/NXI/A116) depicts the flowchart of the patient files reviewed $(\mathrm{n}=37)$ and those included in final analysis $(\mathrm{n}=27)$. The mean age at presentation was $36.6 \pm$ 13.7 (range 8.3-68.1) years, and $85.2 \%(n=23)$ were female. The mean age at presentation for patients with MOG-ON was $41.8 \pm 11.1$ (range 26.2-55.6) years, and $78 \%(\mathrm{n}=7)$ were female. BCVA at nadir revealed no trend toward worst BCVA nadir in the delayed treatment group (4 days as cutoff), with a mean of $1.55 \pm 0.74$ for those treated $>4$ days and $0.99 \pm 0.85$ for those treated $\leq 4$ days, $p=0.085$. It is interesting to note that this trend leveled off to no difference in BCVA nadir when comparing those treated $<7$ days and those treated $\geq 7$ days (BCVA nadir $1.17 \pm 0.83$ in those treated $<7$ days and $1.47 \pm 0.84$ in those treated later, $p=0.41$ ). Patients were treated with IVMP on the same day they presented with ON. The median time to IVMP was 4 days for the whole cohort (range 1-65 days). Of those treated $\geq 7$ days, the median time to IVMP was 21 days (range 9-65 days). The median time to IVMP for those treated earlier than $<7$ days was 3 days and 2 days for those treated within 4 days. Baseline demographic and clinical factors were similar in both the early treatment group ( $<4$ day treatment group) and those treated $>4$ days (percentage of MOG positive $p=0.59$, male $p=0.94$, age $p=$ 0.48 , additional use of plasmapheresis $p=1$ ).

\section{Three-month VA}

There was a significant inverse correlation between BCVA3mo (logMAR) and age $(r=-0.41, p=0.04)$ and days to IVMP treatment $(\mathrm{r}=0.43, p=0.03)$, with a nearly significant correlation between BCVA3mo and logMAR VA at nadir $(r=0.38, p=0.06)$. The distribution of BCVA3mo is depicted in figure 1.

The BCVA3mo was similar between men and women $(0.33 \pm$ 0.52 vs $0.17 \pm 0.47, p=0.61)$ and similar between AQP4positive and MOG-positive patients $(0.11 \pm 0.09$ vs $0.22 \pm$ $0.56, p=0.38)$. Using multivariate analysis, with type of antibody (AQP4 vs MOG), age, days to IVMP treatment, logMAR VA at nadir, and plasmapheresis treatment as the independent variables, the 2 factors that remained significant in predicting BCVA3mo were days to IVMP treatment $(\mathrm{r} 2=$ $15.5 \%, p=0.03)$ and age $(\mathrm{r} 2=16.5 \%, p=0.04)$.

\section{Failure to regain 0.0 logMAR (20/20) vision}

An ROC analysis was performed with days to IVMP treatment as the predictor and failure to regain 0.0 logMAR (20/ $20)$ vision as the dependent variable. An area under the curve (AUC) of 0.71 was achieved (figure 2), and with a Youden optimal criterion of days to treatment $>4$ days, a sensitivity and specificity of $71.4 \%$ and $76.9 \%$, respectively, were achieved. Patients who were treated later than 4 days had an OR of 8.33 (95\% CI 1.47-47.22) of failure to regain 0.0 logMAR vision $(p=0.01)$. The individual AUCs of age and nadir BCVA as individual predictors of failure to regain 0.0 logMAR vision at 3 months were lower ( 0.56 and 0.60 , respectively), and the addition of these 2 predictors to days to IVMP treatment led to a minute improvement in the AUC (0.74) compared with days to treatment alone (0.71).

\section{Failure to regain $0.2 \log M A R$ vision}

\section{( Snellen 20/30)}

A similar analysis with failure to regain $0.2 \log$ MAR as the dependent variable revealed a Youden optimal criterion of days to treatment $>7$ days with an AUC of 0.84 (figure 3), sensitivity of $71.4 \%$, and specificity of $80.0 \%$. Patients treated later than 7 days had an OR of 10.0 (95\% CI 1.39-71.86) of failure to regain $20 / 30$ vision $(p=0.01)$. The individual AUCs of age and nadir BCVA as individual predictors of failure to regain $0.2 \log$ MAR vision at 3 months were lower ( 0.59 and 0.63 , respectively), and the addition of these 2 predictors to days to IVMP treatment led to a reduced AUC (0.80) compared with days to treatment alone (0.84).

\section{Discussion}

In our cohort, patients with AQP4- and MOG-positive ON responded better to earlier IVMP. Of 27 patients with AQP4 or MOG-ON (18 AQP4-IgG+ and 9 MOG-IgG+), and those treated later than 4 days had an OR of 8.33 of failure to regain 20/20 0.0 logMAR vision $(p=0.01)$. Patients treated later than 7 days had an OR of 10.0 of failure to regain 20/30 0.2 $\operatorname{logMAR}$ vision $(p=0.01)$. This finding corroborates a study in patients with acute $\mathrm{ON}$, demonstrating that retinal ganglion cell (RGC) layer loss starts within a few days of ON and may be a predictor of visual loss. ${ }^{32}$

Figure 1 Distribution of BCVA3mo for patients treated with IVMP for AQP4 and MOG-ON

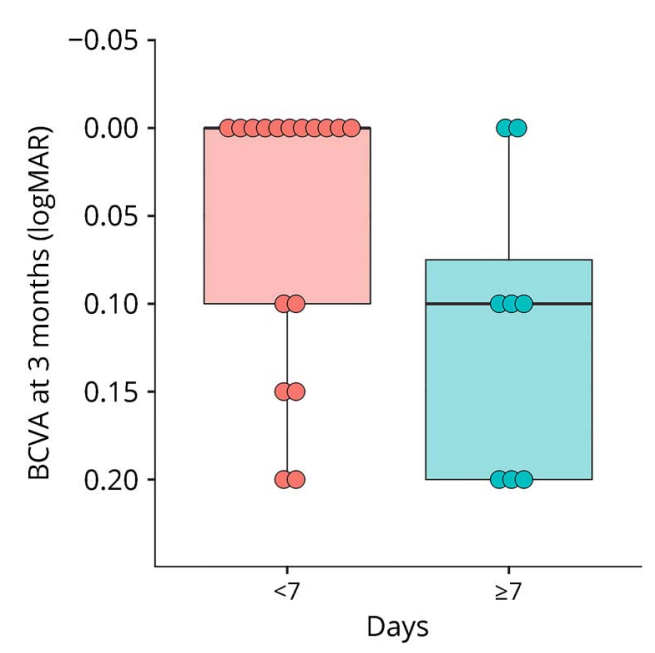

Note inverted logMAR scale: better acuity at top. Left boxplot: eyes of patients treated $<7$ days. Right boxplot: Eyes of patients treated $\geq 7$ days. BCVA3mo = best-corrected visual acuity at 3 months after IVMP treatment for AQP4 and MOG-IgG-ON. Box plot details: thick horizontal bar: median; box: interquartile range (25\%-75\%). Dots: outliers. AQP = aquaporin; $B C V A=$ best-corrected visual acuity; IVMP = IV methylprednisolone; MOG = myelin oligodendrocyte glycoprotein; $\mathrm{ON}=$ optic neuritis. 
Figure 2 A receiver operating characteristic curve of days to IVMP as a predictor of failure to regain 0.0 logMAR $(20 / 20)$ vision (AUC 0.71, $p<0.001$ )

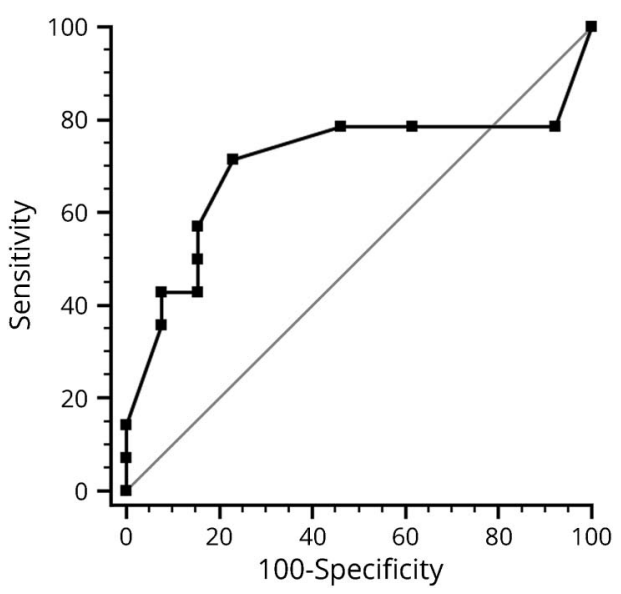

$\mathrm{AUC}=$ area under the curve; IVMP = IV methylprednisolone.

$\mathrm{ON}$ in patients with AQP4-IgG and MOG-IgG antibodies is frequently steroid responsive or dependent, thus differing from MS-ON, in which IVMP does not affect visual outcome. ${ }^{1,33}$ We tested our hypothesis that timing of acute treatment affects visual outcome in AMDD-ON. Despite the small number of patients enrolled and investigated, we were able to construct ROC curves to identify cut points that optimize the balance between sensitivity and specificity in regard to the optimal time window for the administration of IVMP that would also translate into greater improvement of the BCVA at 3 months. Administration of IVMP treatment at day 4 or earlier was the identified cut point $(71.4 \%$ sensitive; $76.9 \%$ specific). Two additional variables affecting visual outcome to a lesser degree were age and VA at nadir.

Offering a better visual outcome for AQP4-seropositive and MOG-seropositive patients with ON implies the need for action in all forms of $\mathrm{ON}$ because at presentation, the etiology is often unclear. In MS-related ON, the ONTT suggested that final visual outcome is not affected by acute treatment with IVMP,${ }^{15,16}$ leading to a sense of nonurgency in the acute phase of ON. A change of treatment paradigm, especially in acceleration of IVMP timing in acute ON treatment, may be needed. For a significant number of patients who harbor AQP4 $a b$ or MOG ab at presentation, it may be crucial to start IVMP treatment for $\mathrm{ON}$ as soon as possible.

Perhaps most salient about this submission was the recognition that as little as a 7-day delay in treatment inception (for NMOSD and anti-MOG-associated optic neuritides) was found to be detrimental in terms of the OR for improving BCVA at 3 months after symptom onset. "Time is Tissue" is a core principal that is evolving in the field of neuroimmunology, ${ }^{34}$ making it imperative to potentially view an antibody-mediated ON with a comparable sense of urgency in terms of diagnosis and treatment akin to that of heart attack and stroke. Our findings are in good alignment with the findings by Soelberg et al., ${ }^{32}$ who reported that in ON, the majority of which were not antibody mediated, progressive ganglion cell layer loss at a rate of $0.2 \mu \mathrm{m} / \mathrm{d}$ can be observed as early as 8 days after onset.

Corroborating the contention of Time is Tissue has been the recognition of inflammation as a fundamental antecedent of the cardinal hallmark of irreversible disability in those with inflammatory syndromes of the CNS; that being axonal transection, the evolution of dying back and Wallerian degeneration. Among the most striking observations of this proposed model of sequential steps in the pathobiology of postinflammatory neurodegeneration has been the degeneration of RGCs within a time epoch as short as 2 days of the onset of clinical symptoms ultimately designated as a derivative of such inflammation.

The results of our study strengthen 3 previous reports in other forms of $\mathrm{ON}^{23-25}$ demonstrating a beneficial effect of hyperacute IVMP. These studies ${ }^{23-25}$ did not focus on AQP4 and MOG-ON; Osinga et al. ${ }^{23}$ described a cohort of 19 patients with recurrent $\mathrm{ON}, 9$ of whom with relapsing isolated ON, 4 with MS-ON, 4 with chronic relapsing inflammatory optic neuropathy, and 2 with NMOSD-ON. These 19 patients were analyzed for the effects of treatment within 2 days (hyperacute treatment). The importance of hyperacute steroids in ON treatment has experimental logic in animal models. In mice with experimental autoimmune encephalomyelitis, the inflammatory process precedes axonal degeneration by 2 days. ${ }^{35} \mathrm{~A}$ goal of treatment within 2 days of symptom onset is difficult to achieve in clinical reality. Another study by Zhu et al. ${ }^{36}$ showed that irreversible axonal damage starts between days 5 and 7, supporting our clinical

Figure 3 A receiver operating characteristic curve of days to IVMP as a predictor of failure to regain $0.2 \log M A R$ (20/30) vision (AUC 0.84, $p<0.001$ )

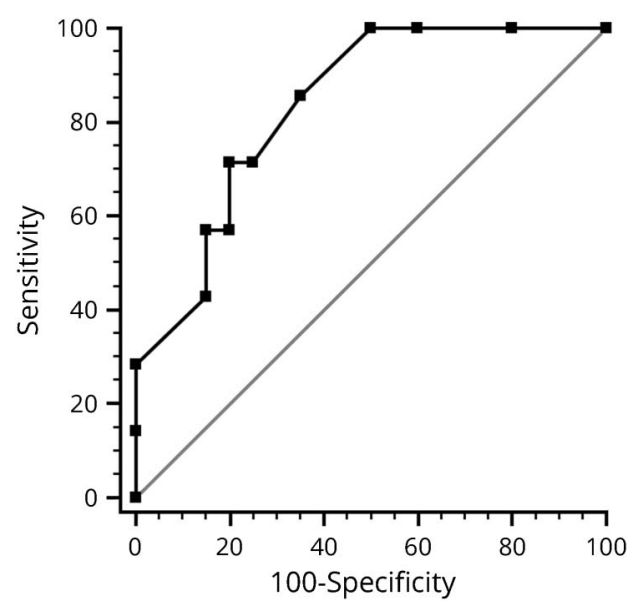

AUC = area under the curve; IVMP = IV methylprednisolone. 
finding that optimal treatment is by day 4, but that treatment before day 7 still offers an opportunity for very good visual outcome.

Previous MRI and OCT studies have demonstrated that the bulk of axonal loss and neuronal damage is sustained early in the disease course for patients with MS. ${ }^{37,38}$ Although the rates of ganglion cell-inner plexiform layer atrophy may be influenced by disease-modifying therapies in patients with MS, further studies, using the detailed structural OCT tools currently at our disposal, should re-examine the effect of timing of IVMP on visual outcome in other forms of $\mathrm{ON}$.

Few clinical studies on outcome of NMOSD-ON include details of accurate timing from symptom onset to acute treatment, and there is much need for this detail to be analyzed in larger cohorts. The results of this study show a trend indicating that even a 7-day delay in IVMP can be detrimental to vision in AQP4 and MOG-IgG ON. Several limitations should be taken into consideration when considering these results, including the study's retrospective design, the small sample size resulting in very large confidence intervals, the short follow-up duration, and the lack of paraclinical data to confirm the functional results with structural indices such as loss of retinal nerve fiber and ganglion cell layers on OCT.

A prospective study in a larger cohort of patients with NMOSD examining the effects of timing of IVMP on additional visual parameters such as OCT, visual fields BCVA, and on subsequent $\mathrm{ON}$ attacks seems warranted.

\section{Study funding}

No targeted funding reported.

\section{Disclosure}

H. Kalish received research support from the Maratier Foundation, Tel Aviv University, and Israeli Car Accident Prevention Association. M.A. Hellmann and M. Mimouni report no disclosures. F. Paul served on the scientific advisory boards of Novartis and MedImmune; received speaker honoraria and travel funding from Bayer, Novartis, Biogen, Teva, Sanofi-Aventis/Genzyme, Merck Serono, Alexion, Chugai, MedImmune, and Shire; serves as academic editor of PLoS ONE and associate editor of Neurology: Neuroimmunology \& Neuroinflammation; consulted for Sanofi-Genzyme, Biogen, MedImmune, Shire, and Alexion; and received research support from Bayer, Novartis, Biogen, Teva, Sanofi-Aventis/ Genzyme, Alexion, Merck Serono, German Research Council, Werth Stiftung of the City of Cologne, German Ministry of Education and Research, Arthur Arnstein Stiftung Berlin, EU FP7 Framework Program, Guthy Jackson Charitable Foundation, and NMSS. O. Bialer and M. Bach report no disclosures. L. Lotan received travel funding from Teva, Merck Serono, Biogen, and Sanofi-Genzyme. Full disclosure form information provided by the authors is available with the full text of this article at Neurology.org/NN.

\section{Publication history}

Received by Neurology: Neuroimmunology \& Neuroinflammation December 10, 2018. Accepted in final form March 8, 2019.

Appendix Authors

\begin{tabular}{llll}
\hline Name & Location & Role & Contribution \\
\hline $\begin{array}{l}\text { Hadas } \\
\text { Stiebel- } \\
\text { Kalish, MD }\end{array}$ & $\begin{array}{l}\text { Tel Aviv University \& } \\
\text { Rabin Medical Ctr. }\end{array}$ & Author & $\begin{array}{l}\text { Designed and } \\
\text { conceptualized the } \\
\text { study, analyzed the } \\
\text { data, and drafted } \\
\text { the manuscript for } \\
\text { intellectual content }\end{array}$ \\
$\begin{array}{l}\text { Mark } \\
\text { Andrew } \\
\text { Hellmann, } \\
\text { MD }\end{array}$ & Tel Aviv University \& & Author & $\begin{array}{l}\text { Major role in the } \\
\text { acquisition of data } \\
\text { and revised the } \\
\text { manuscript for }\end{array}$ \\
& & & $\begin{array}{l}\text { intellectual content } \\
\text { ind Ctr. }\end{array}$
\end{tabular}

\begin{tabular}{|c|c|c|c|}
\hline $\begin{array}{l}\text { Michael } \\
\text { Mimouni, } \\
\text { MD }\end{array}$ & $\begin{array}{l}\text { Rambam HCC, \& } \\
\text { Technion-Israel } \\
\text { Institute of } \\
\text { Technology }\end{array}$ & Author & $\begin{array}{l}\text { Interpreted the data, } \\
\text { statistical analysis, } \\
\text { and revised the } \\
\text { manuscript for } \\
\text { intellectual content }\end{array}$ \\
\hline $\begin{array}{l}\text { Friedemann } \\
\text { Paul, MD }\end{array}$ & $\begin{array}{l}\text { NeuroCure, Charité } \\
\text { Universitätsmedizin } \\
\text { Berlin, Freie } \\
\text { Universität Berlin, } \\
\text { Humboldt-Universität } \\
\text { zu Berlin, \& Berlin } \\
\text { Institute of Health, } \\
\text { Berlin, Germany }\end{array}$ & Author & $\begin{array}{l}\text { Interpreted the data } \\
\text { and revised the } \\
\text { manuscript for } \\
\text { intellectual content }\end{array}$ \\
\hline $\begin{array}{l}\text { Omer Bialer, } \\
\text { MD }\end{array}$ & $\begin{array}{l}\text { Tel Aviv University \& } \\
\text { Rabin Medical Ctr. }\end{array}$ & Author & $\begin{array}{l}\text { Major role in the } \\
\text { acquisition of data } \\
\text { and revised the } \\
\text { manuscript for } \\
\text { intellectual content }\end{array}$ \\
\hline $\begin{array}{l}\text { Michael } \\
\text { Bach, PhD }\end{array}$ & $\begin{array}{l}\text { University of Freiburg, } \\
\text { Germany \& Faculty of } \\
\text { Medicine, Germany }\end{array}$ & Author & $\begin{array}{l}\text { Interpreted the data, } \\
\text { graphical and } \\
\text { statistical analysis, } \\
\text { and revised the } \\
\text { manuscript for } \\
\text { intellectual content }\end{array}$ \\
\hline $\begin{array}{l}\text { Itay Lotan, } \\
\text { MD }\end{array}$ & $\begin{array}{l}\text { Tel Aviv University \& } \\
\text { Rabin Medical Ctr. }\end{array}$ & Author & $\begin{array}{l}\text { Major role in the } \\
\text { acquisition of data } \\
\text { and revised the } \\
\text { manuscript for } \\
\text { intellectual content }\end{array}$ \\
\hline
\end{tabular}

\section{References}

1. Balcer LJ. Clinical practice. Optic neuritis. N Engl J Med 2006;354:1273-1280.

2. Galetta SL, Villoslada P, Levin N, et al. Acute optic neuritis: unmet clinical needs and model for new therapies. Neurol Neuroimmunol Neuroinflamm 2015;2:e135. doi:10. 1212/NXI.0000000000000135.

3. Soelberg K, Jarius S, Skejoe HPB, et al. A population-based prospective study of optic neuritis. Mult Scler J 2017;23:1893-1901.

4. Petzold A, Wattjes MP, Costello F, et al. The investigation of acute optic neuritis: a review and proposed protocol. Nat Rev Neurol 2014;10:447-458.

5. Atkins EJ. Optic neuritis. Encycl Neurol Sci 2014;13:681-686.

6. Wingerchuk DM, Banwell B, Bennett JL, et al. International consensus diagnostic criteria for neuromyelitis optica spectrum disorders. Neurology 2015;85:177-189.

7. Jarius S, Kleiter I, Ruprecht K, et al. MOG-IgG in NMO and related disorders: a multicenter study. Part 3: mOG-IgG-associated brainstem encephalitis. Mult Scler 2016; Conference: 410-411. Available at: cochranelibrary.com/central/doi/10.1002/central/CN-01212258/full.

8. Biotti D, Bonneville F, Tournaire E, et al. Optic neuritis in patients with anti-MOG antibodies spectrum disorder: MRI and clinical features from a large multicentric cohort in France. J Neurol 2017;264:2173-2175.

9. Ramanathan S, Dale RC, Brilot F. Anti-MOG antibody: the history, clinical phenotype, and pathogenicity of a serum biomarker for demyelination. Autoimmun Rev 2016;15:307-324. 
10. Akaishi T, Sato DK, Takahashi T, Nakashima I. Clinical spectrum of inflammatory central nervous system demyelinating disorders associated with antibodies against myelin oligodendrocyte glycoprotein. Neurochem Int Epub 2018 Oct 23.

11. Chalmoukou K, Alexopoulos H, Akrivou S, Stathopoulos P, Reindl M, Dalakas MC Clinical/scientific notes. Neurol Neuroimmunol Neuroinflamm 2015;2:1-3.

12. Borisow N, Mori M, Kuwabara S, Scheel M, Paul F. Diagnosis and treatment of NMO spectrum disorder and MOG-encephalomyelitis. Front Neurol 2018;9:888.

13. Schmidt F, Zimmermann H, Mikolajczak J, et al. Severe structural and functional visual system damage leads to profound loss of vision-related quality of life in patients with neuromyelitis optica spectrum disorders. Mult Scler Relat Disord 2017;11: 45-50.

14. Zamvil SS, Slavin AJ. Does MOG Ig-positive AQP4-seronegative opticospinal inflammatory disease justify a diagnosis of NMO spectrum disorder? Neurol Neuroimmunol Neuroinflamm 2015;2:e62. doi:10.1212/NXI.0000000000000062.

15. Beck RW. The optic neuritis treatment trial: three-year follow-up results. Arch Ophthalmol 1995;113:136-137.

16. Kupersmith MJ, Anderson S, Kardon R. Predictive value of 1 month retinal nerve fiber layer thinning for deficits at 6 months after acute optic neuritis. Mult Scler J 2013;19: 1743-1748.

17. Pittock SJ, Lucchinetti CF. Neuromyelitis optica and the evolving spectrum of autoimmune aquaporin-4 channelopathies: a decade later. Ann N Y Acad Sci 2016;1366: 20-39.

18. Sato D, Callegaro D, Lana-Peixoto MA, Fujihara K; Brazilian Committee for Treatment and Research in Multiple Sclerosis. Treatment of neuromyelitis optica: an evidence based review. Arq Neuropsiquiatr 2012;70:59-66.

19. Kleiter I, Gahlen A, Borisow N, et al. Apheresis therapies for NMOSD attacks: a retrospective study of 207 therapeutic interventions criteria for rating therapeutic and diagnostic studies. Neurol Neuroimmunol Neuroinflamm 2018;5:e504. doi:10. 1212/NXI.0000000000000504

20. Wingerchuk DM, Hogancamp WF, O'Brien PC, Weinshenker BG. The clinical course of neuromyelitis optica (Devic's syndrome). Neurology 1999;53:1107.

21. Bonnan M, Valentino R, Debeugny S, et al. Short delay to initiate plasma exchange is the strongest predictor of outcome in severe attacks of NMO spectrum disorders. J Neurol Neurosurg Psychiatry 2018;89:346-351.

22. Mori S, Kurimoto T, Ueda K, Nakamura M. Short-term effect of additional apheresis on visual acuity changes in patients with steroid-resistant optic neuritis in neuromyelitis optica spectrum disorders. Jpn J Ophthalmol 2018;62:525-530.
23. Osinga E, van Oosten B, de Vries-Knoppert W, Petzold A. Time is vision in recurrent optic neuritis. Brain Res 2017;1673:95-101.

24. Plant GT, Sibtain NA, Thomas D. Hyperacute corticosteroid treatment of optic neuritis at the onset of pain may prevent visual loss: a case series. Mult Scler Int 2011; 2011:815068.

25. Nakamura M, Nakazawa T, Doi H, et al. Early high-dose intravenous methylprednisolone is effective in preserving retinal nerve fiber layer thickness in patients with neuromyelitis optica. Graefe's Arch Clin Exp Ophthalmol 2010;248:1777-1785.

26. Filippi M, Rocca MA, Ciccarelli O, et al. MRI criteria for the diagnosis of multiple sclerosis: MAGNIMS consensus guidelines. Lancet Neurol 2016;15:292-303.

27. Balcer LJ, Miller DH, Reingold SC, Cohen JA. Vision and vision-related outcome measures in multiple sclerosis. Brain 2015;138(pt 1):11-27.

28. Jarius S, Paul F, Aktas O, et al. MOG encephalomyelitis: international recommendations on diagnosis and antibody testing [in German]. Nervenarzt 2018;89:1388-1399.

29. Höftberger R, Sabater L, Marignier R, et al. An optimized immunohistochemistry technique improves NMO-IgG detection: study comparison with cell-based assays. PLoS One 2013;8:6-11.

30. Höftberger R, Sepulveda M, Armangue T, et al. Antibodies to MOG and AQP4 in adults with neuromyelitis optica and suspected limited forms of the disease. Mult Scler 2015;21:866-874.

31. Youden WJ. Index for rating diagnostic tests. Cancer 1950;3:32-35.

32. Soelberg K, Specovius S, Zimmermann HG, et al. Optical coherence tomography in acute optic neuritis: a population-based study. Acta Neurol Scand 2018;138:566-573.

33. Group RWB and the optic neuritis study; Beck RW, Cleary PA, Anderson MM Jr, et al. A randomized, controlled trial of corticosteroids in the treatment of acute optic neuritis. The Optic Neuritis Study Group. N Engl J Med 1992;326:581-588.

34. Eshaghi A, Prados F, Brownlee WJ, et al. Deep gray matter volume loss drives disability worsening in multiple sclerosis. Ann Neurol 2018;83:210-222.

35. Shindler KS, Ventura E, Dutt M, Rostami A. Inflammatory demyelination induces axonal injury and retinal ganglion cell apoptosis in experimental optic neuritis. Exp Eye Res 2008;87:208-213.

36. Zhu B, Moore GRW, Zwimpfer TJ, et al. Axonal cytoskeleton changes in experimental optic neuritis. Brain Res 1999;824:204-217.

37. Balk LJ, Cruz-Herranz A, Albrecht P, et al. Timing of retinal neuronal and axonal loss in MS: a longitudinal OCT study. J Neurol 2016;263:1323-1331.

38. Granberg T, Fan Q, Treaba CA, et al. In vivo characterization of cortical and white matter neuroaxonal pathology in early multiple sclerosis. Brain 2017;140:2912-2926. 


\section{Neurology \\ Neuroimmunology \& Neuroinflammation}

\section{Does time equal vision in the acute treatment of a cohort of AQP4 and MOG optic neuritis?}

Hadas Stiebel-Kalish, Mark Andrew Hellmann, Michael Mimouni, et al.

Neurol Neuroimmunol Neuroinflamm 2019;6;

DOI 10.1212/NXI.0000000000000572

This information is current as of May 21, 2019

\section{Updated Information \& Services}

References

Citations

Subspecialty Collections

Permissions \& Licensing

Reprints including high resolution figures, can be found at: http://nn.neurology.org/content/6/4/e572.full.html

This article cites 36 articles, 1 of which you can access for free at: http://nn.neurology.org/content/6/4/e572.full.html\#\#ref-list-1

This article has been cited by 19 HighWire-hosted articles: http://nn.neurology.org/content/6/4/e572.full.html\#\#otherarticles

This article, along with others on similar topics, appears in the following collection(s):

Devic's syndrome

http://nn.neurology.org//cgi/collection/devics_syndrome Optic neuritis; see Neuro-ophthalmology/Optic Nerve http://nn.neurology.org//cgi/collection/optic_neuritis

Information about reproducing this article in parts (figures,tables) or in its entirety can be found online at:

http://nn.neurology.org/misc/about.xhtml\#permissions

Information about ordering reprints can be found online: http://nn.neurology.org/misc/addir.xhtml\#reprintsus

Neurol Neuroimmunol Neuroinflamm is an official journal of the American Academy of Neurology.

Published since April 2014, it is an open-access, online-only, continuous publication journal. Copyright Copyright (C) 2019 The Author(s). Published by Wolters Kluwer Health, Inc. on behalf of the American Academy of Neurology.. All rights reserved. Online ISSN: 2332-7812.

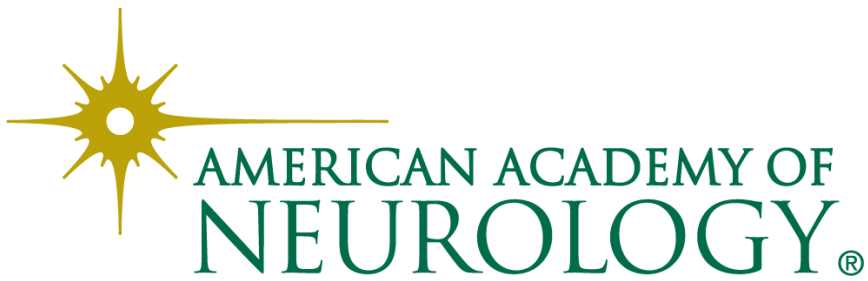

